\title{
SYMBOLOGY DEVELOPMENT FOR GENERAL AVIATION SYNTHETIC VISION PRIMARY FLIGHT DISPLAYS FOR THE APPROACH AND MISSED-APPROACH MODES OF FLIGHT
}

\author{
Anthony P. Bartolone, Monica F. Hughes, Douglas T. Wong \\ NASA Langley Research Center \\ Hampton, VA \\ Mohammad A. Takallu \\ Lockheed Martin Corporation \\ Hampton, VA
}

\begin{abstract}
Spatial disorientation induced by inadvertent flight into instrument meteorological conditions (IMC) continues to be a leading cause of fatal accidents in general aviation. The Synthetic Vision Systems General Aviation (SVS-GA) research element, an integral part of NASA's Aviation Safety and Security Program (AvSSP), is investigating a revolutionary display technology designed to mitigate low visibility events such as controlled flight into terrain (CFIT) and low-visibility loss of control (LVLoC). The integrated SVS Primary Flight Display (SVS-PFD) utilizes computer generated 3-dimensional imagery of the surrounding terrain augmented with flight path guidance symbology. This unique combination will provide GA pilots with an accurate representation of their environment and projection of their flight path, regardless of time of day or out-the-window (OTW) visibility. The initial Symbology Development for Head-Down Displays (SD-HDD) simulation experiment examined 16 display configurations on a centrally located high-resolution PFD installed in NASA's General Aviation Work Station (GAWS) flight simulator. The results of the experiment indicate that situation awareness (SA) can be enhanced without having a negative impact on flight technical error (FTE), by providing a general aviation pilot with an integrated SVS display to use when OTW visibility is obscured.
\end{abstract}

\section{INTRODUCTION}

Spatial disorientation induced by inadvertent flight into instrument meteorological conditions (IMC) continues to be a leading cause of fatal accidents in general aviation (GA). The Aircraft Owners \& Pilots Association's (AOPA) 2002 Nall Report, a review of the previous year's general aviation accidents, stated that accidents that resulted from attempted VFR flight into IMC were fatal $84 \%$ of the time (Hummel, Landsberg, Roy, \& Wright, 2002). At present, an immediate exit from IMC is the only recourse a VFR pilot has to avoid the perils that accompany the loss of out-the-window (OTW) visibility. The Synthetic Vision Systems - General Aviation (SVS-GA) research element, an integral part of NASA's Aviation Safety and Security Program (AvSSP), is investigating a revolutionary display technology designed to mitigate such events. This research, a cooperative effort with various industry, university, and federal agency, partners is dedicated to the goal of developing an affordable system that will drastically reduce the occurrence of controlled flight into terrain (CFIT) and low-visibility loss of control (LVLoC) accidents.

SVS technology provides a visual solution to a visibility problem. The integrated SVS Primary Flight Display (PFD) utilizes computer generated 3-dimensional imagery of the surrounding terrain and obstacles. When SVS terrain is coupled with flight path guidance symbology, this unique combination provides GA pilots with an accurate headdown representation of their current operating environment and a projection of their flight path, regardless of time of day or OTW visibility, thereby greatly increasing situation awareness (SA) over conventional instrumentation. SVS systems are designed to provide the pilot with a perspective view that is highly intuitive on a display that is congruent with the pilot's natural mode of spatial information gathering. The results of the Terrain Portrayal for Head Down Displays (TPHDD) experiment and flight test, a precursor to the current SVS-GA research, had shown these statements to be valid (Hughes and Glaab, 2003; Glaab and Hughes, 2003). Testing the interaction between Terrain Portrayal Concepts (TPC) and Guidance Symbology Concepts (GSC) on the PFD is the focus of the Symbology Development for Head Down Displays (SD-HDD) series of flight simulator experiments, the latest series of SVS-GA research experiments.

\section{METHOD}

Several hypotheses were developed for the SD-HDD experiment. The first was that adding terrain to the PFD would improve pilot SA across all guidance symbology concepts. Second, low-fidelity (simple) terrain concepts will favor complex guidance symbology (Connected Box Tunnel or Tunnel with Ghost Aircraft). Third, high-fidelity terrain concepts will favor simple guidance symbology (Pitch/Roll 
Flight Director (P/RFD) or Unconnected Box Tunnel). Finally, pilot's technical performance will be improved with the tunnel concepts without increasing pilot workload.

The experiment was conducted at NASA Langley Research Center (LaRC) using the SVS-GA General Aviation WorkStation (GAWS) fixed-base flight simulator. GAWS utilizes a Precision Flight Controls PC-based Aviation Training Device model PI-142 instrument trainer coupled with two 6-inch VGA monitors on the instrument panel and a SXGA overhead projector for the OTW depiction. For the SD-HDD experiment, the evaluation pilot (EP) flew the scenarios from the left seat and was required to utilize the yoke, rudder pedals, and throttle quadrant, to operate the simulator. The primary elements of the GAWS instrument panel included the SVS-PFD and a navigation display (ND) with multi-level range selection capability, developed at NASA LaRC. Pilot selectable fields of view (FOV) (horizontal angle of the image that is presented on the display) of 30 and 60 degrees were available throughout the scenarios for the PFD and the boundaries of the PFD FOV were depicted on the ND. Finally, the Initiative Computing Electronic Instrument Training Environment (ELITE) flight simulator software was configured with a Cessna C-172 dynamic flight model.

This experiment, the initial simulation experiment of the SD-HDD series, focused on the approach and missedapproach modes of flight. In addition to the current basic PFD background of Blue Sky over Brown Ground (BSBG), three Terrain Portrayal Concepts (TPC) were chosen (Figure 1) for the SVS experimental terrain database. Constant Color with Fish Net (CCFN), the lowest fidelity terrain concept, used a single color as the terrain texture and included a consistently spaced overlaying grid for the elevation cues of a digital elevation model (DEM) with a resolution of $60 \mathrm{arc} / \mathrm{sec}$. The medium-fidelity terrain concept, Elevation Based Generic (EBG), utilized 12 graduated color bands to depict elevation gradients within its 6 arc/sec DEM resolution database. Green was used to depict field elevation; the highest terrain within 50 nm was colored white; and uniform height color bands were used for elevations in between for the EBG concept. Photo Realistic (PR), the highest fidelity terrain concept, used digital satellite photographs (4-meter imagery) overlaid on a 2 arc/sec DEM resolution database. The Guidance Symbology Concepts (GSC) chosen for the experiment were three tunnel concepts (Tunnel with Ghost Aircraft, Unconnected Box Tunnel, and Connected Box Tunnel) and one dual-cue flight director (Pitch/Roll Flight Director) (Figure 2). The dual-cue flight director served as the baseline for GSC for this experiment as this concept is used for current day PFD-type displays. Each of the tunnel concepts were adopted from various research organizations and industry applications and represent specific concepts in this area. Parameters that define each tunnel concept, such as size, shape, color, etc., were not modified from their nominal values for each tunnel concept. The tunnel concepts were also selected due to their relative clutter characteristics (from low to high). Furthermore, three of the four GSC automatically altered their attributes when the pilot executed a missed-approach. The pitch cue on the Pitch/Roll Flight Director was modified to become a speed- on-pitch command, while the upper vertical limits were removed from the Tunnel with Ghost Aircraft and Connected Box Tunnel, thereby encouraging the pilot to gain altitude while executing a missed approach.

\begin{tabular}{cccc}
\hline $\begin{array}{c}\text { Pilot } \\
\text { Category }\end{array}$ & N & $\begin{array}{c}\text { Mean } \\
\text { Age } \\
\text { (yrs) }\end{array}$ & $\begin{array}{c}\text { Mean } \\
\text { Experience } \\
\text { (hrs) }\end{array}$ \\
\hline VFR & 9 & 43 & 293 \\
IFR & 6 & 35 & 766 \\
H-IFR & 6 & 53 & 11,632 \\
\hline Total & 21 & 44 & 3,668 \\
\hline
\end{tabular}

Table 1. Evaluation Pilot (EP) Data

The four TPC and four GSC combined to make a total of sixteen experimental display configurations examined for both the approach and missed-approach scenarios over the course of a two-day within subject GAWS simulator experiment. Twenty-one subjects ranging in experience from low-time VFR pilots to high-time IFR pilots (Table 1) participated in the SD-HDD experiment. The Juneau, Alaska, terminal area (JNU) served as the simulation area of operations for the two distinct scenarios. The approach scenario required the subject pilot to fly downwind, base, and short- final, flight segments to Runway 26, using a VMC-like approach for each of the 16 SD-HDD display concepts. Similarly, the missed-approach scenario required the subject pilot to fly short-final, straight-climb missed-approach, climbing right-turn crosswind, and downwind, flight segments for Runway 8 at JNU, using each of the 16 SD-HDD display concepts. For both scenarios, OTW visibility decreased from 9 nautical miles (nm) to $1 \mathrm{~nm}$ within one minute of the beginning of each data collection run.

\section{RESULTS}

The dependent variables for this SD-HDD experiment were both objective and subjective. For the objective data measures, a real-time data processor was used to score each data collection run immediately after data collection for that run finished. A numerical score for each run was calculated in terms of percent of time within the Level-1 (L1) flight technical performance category based on the combination of airspeed, vertical path, and lateral path, error. The subjective data measures included a 3-D Situation Awareness Rating Technique (SART), a standard NASA-TLX (TLX) workload index, and specific questions about terrain awareness and guidance awareness. At the end of each data collection run the subject pilot was required to complete the three-page subjective run questionnaire, administered digitally on a Tablet PC. The run questionnaire provided the EP's with the ability to convey instantaneous subjective estimates of SA (SART) and perceived workload (NASA-TLX) for each of the 16 display concepts in both the approach and missed-approach scenarios, immediately after exposure to each particular display concept. Both the objective and subjective data collected during the SD-HDD experiment were statistically analyzed using SPSS software. 

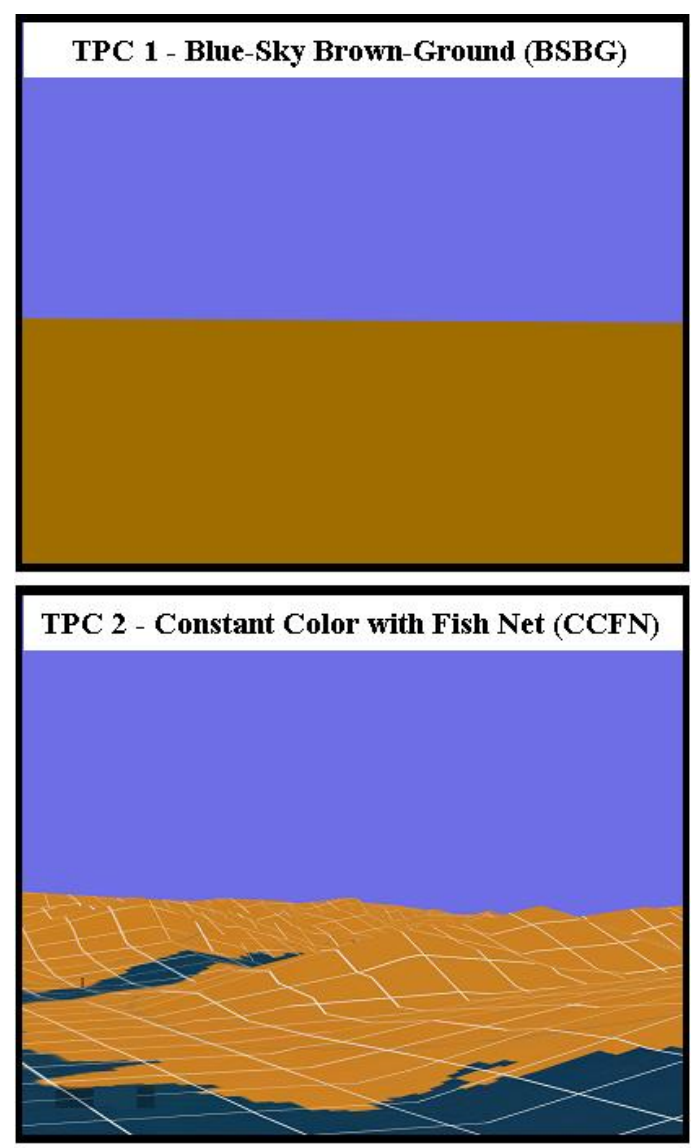

TPC 3 - Elevation Based Generic (EBG)

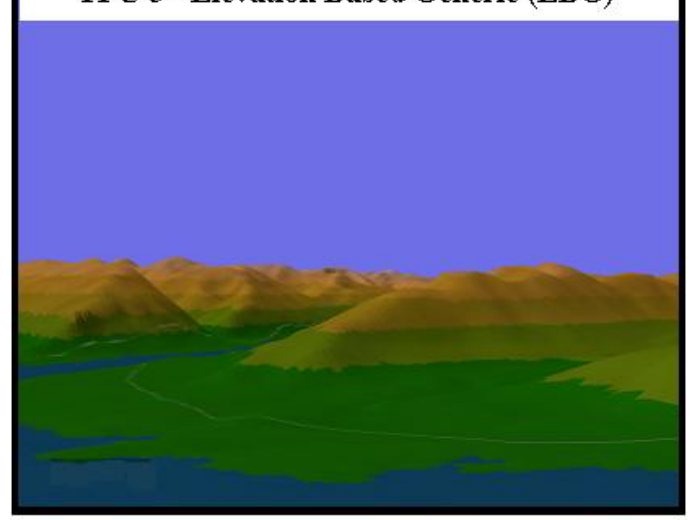

TPC 4 - Photo Realistic (PR)

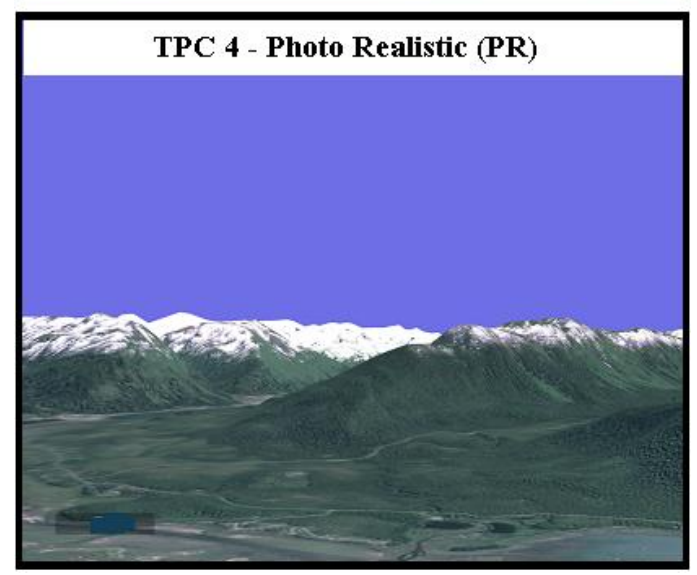

Figure 1 - Terrain Portrayal Concepts (TPC)
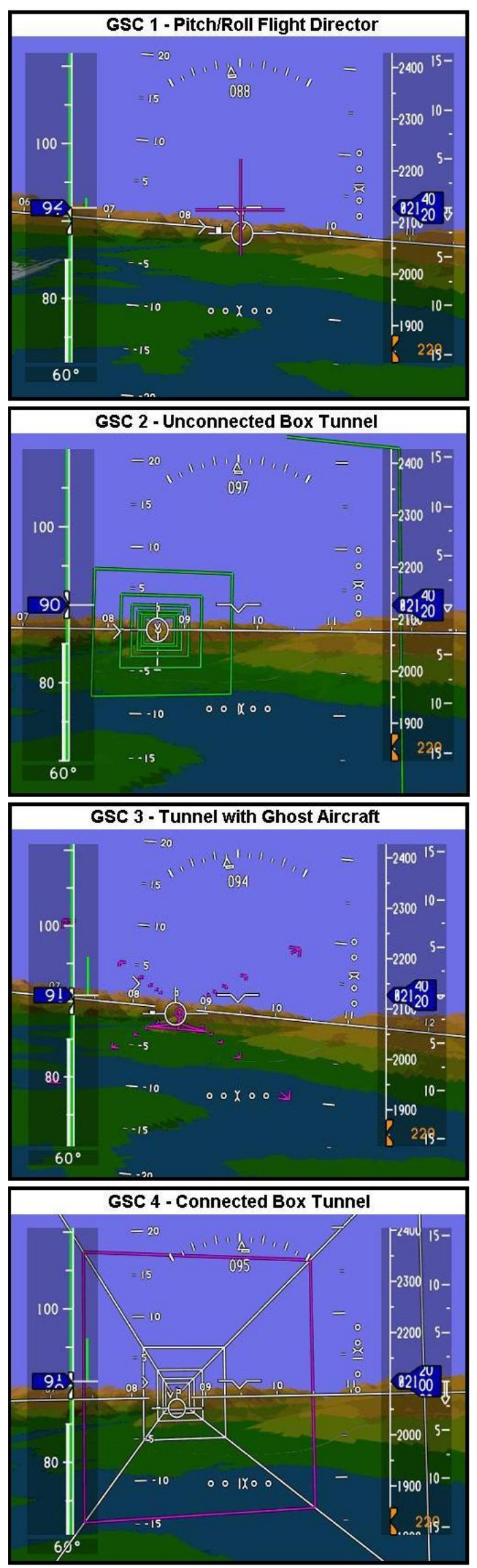

Figure 2. Guidance Symbology Concepts (GSC) 


\section{Workload}

A significant effect was found for GSC with respect to perceived workload (NASA-TLX), F $(3,332)=7.995(\mathrm{p}$ $<.05$ ), for the missed-approach scenario (Figure 3a). The tunnel with ghost aircraft GSC was found to produce significantly less workload than the other concepts, for the missed-approach scenario. There were no significant differences for GSC with respect to workload for the approach scenario and TPC was not significant for either scenario.
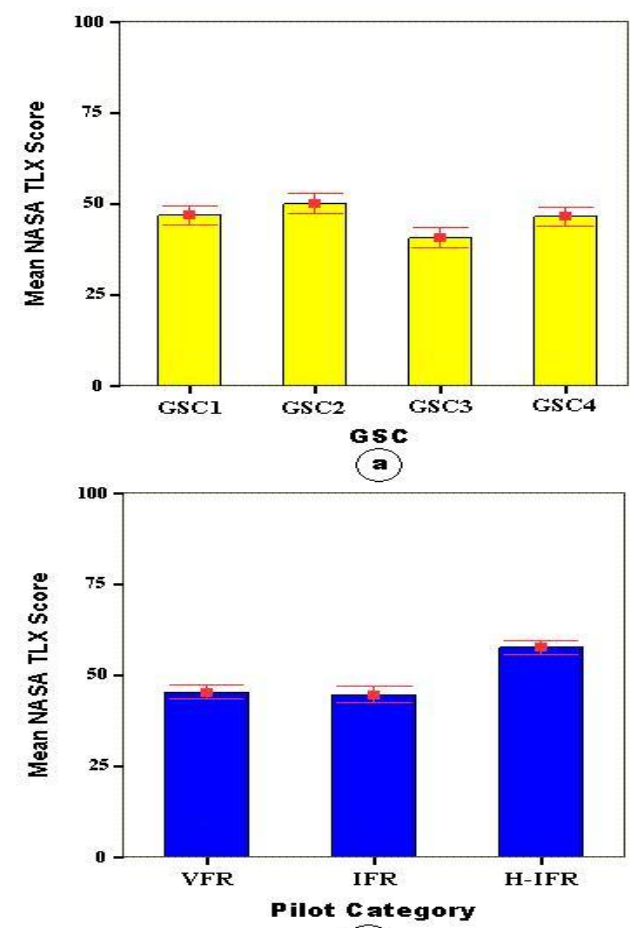

(b)

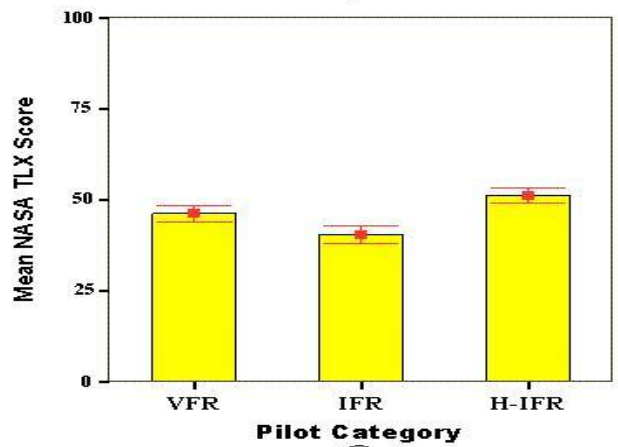

(c)

Figure 3. Mean NASA TLX Scores

There was a significant effect found for pilot category with respect to workload for the approach scenario, $F(2,333)=$ 44.361 ( $\mathrm{p}<.05)$ (Figure 3b), as well as for the missedapproach scenario, $\mathrm{F}(2,333)=18.434(\mathrm{p}<.05)($ Figure 3c). In both scenarios, high-time IFR (H-IFR) pilots were found to have the highest perceived workload. There were no significant differences found for either the approach or missed-approach scenarios for the interaction of TPC and GSC with respect to perceived workload.

\section{Situation Awareness}

A significant effect was found for GSC with respect to SA, F $(3,332)=13.962(p<.05)$, for the missed-approach scenario. The tunnel with ghost aircraft GSC was found to produce significantly higher subjective SA then the other concepts, for the missed-approach scenario. There were no significant differences for GSC with respect to subjective SA for the approach scenario. There was a significant effect found for TPC with respect to SA for the approach scenario,

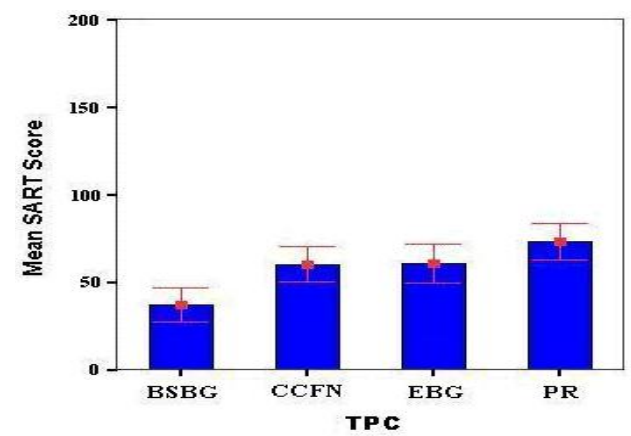

(a)
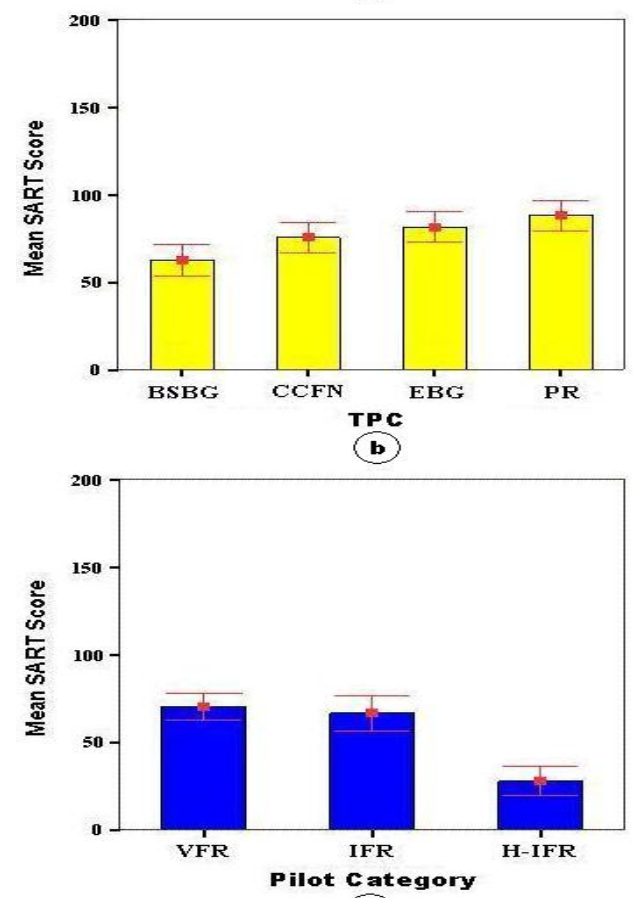

(c)

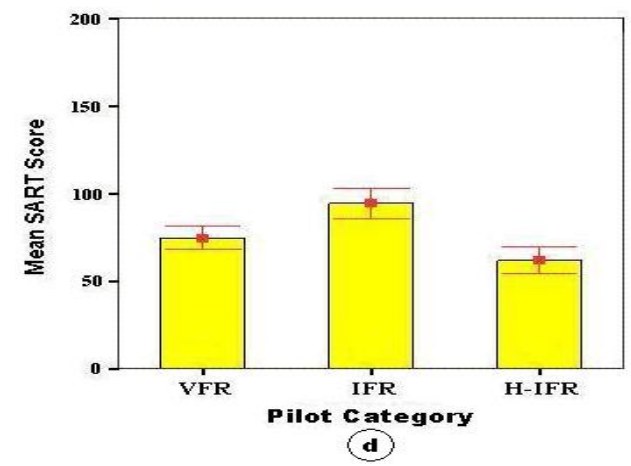

Figure 4. Mean SART Scores 
$F(2,332)=8.156(p<.05)$ (Figure 4a), as well as for the missed-approach scenario, F $(2,332)=5.960(p<.05)$ (Figure $4 \mathrm{~b})$. In both scenarios, the baseline PFD background of BSBG was found to have the lowest subjective SA. There were no significant differences found for either the approach or missed-approach scenarios for the interaction of TPC and GSC with respect to subjective SA. There was a significant effect found to exist for pilot category with respect to subjective SA (SART) for the approach scenario, $\mathrm{F}(2,333)=$ 27.666 ( $<$ <.05) (Figure 4c), and for the missed-approach scenario, $F(2,333)=16.203(p<.05)$ (Figure 4d). In both scenarios, high-time IFR (H-IFR) pilots were found to have the lowest subjective SA.

\section{Level-1 Performance}

Immediately following each data collection run, a numerical score for evaluation pilots were calculated in terms of percent of time within the Level-1 (L1) flight technical performance category. L1 performance was analyzed for both the approach and missed-approach scenarios however vertical path error was not a graded measure for the missed-approach scenario. An ANOVA performed on the L1 data found a significant effect for GSC on L1 performance for the approach scenario, $F(3,320)=24.842(p<.01)$, as well as for the missed-approach scenario, $\mathrm{F}(3,287)=22.407(\mathrm{p}<.01)$. The Tunnel with Ghost Aircraft GSC was found to be significantly better for achieving L1 performance for both scenarios. There were no significant differences found for TPC or the interaction of TPC and GSC with respect to L1 performance for either the approach or missed-approach scenarios. Furthermore, there were no significant differences found for pilot category with respect to L1 performance for the approach scenario, however, pilot category did have a significant effect on L1 performance for the missed-approach scenario, $F(2$, $287)=3.9(\mathrm{P}<0.02)$. The H-IFR pilot group had a slightly higher mean L1 performance then the other pilot groups for the missed-approach scenario.

\section{DISCUSSION}

The first experiment hypothesis stated that providing terrain on the PFD would improve pilot SA across all guidance symbology concepts. The results above indicate that situation awareness (SA) was enhanced without negatively impacting pilot workload or flight technical error (FTE) during the approach and missed approach modes of flight, by providing a general aviation pilot with an integrated SVS display to use when OTW visibility is obscured. The second and third hypotheses stated that low fidelity (simple) terrain concepts would favor complex guidance symbology (Connected Box Tunnel or Tunnel with Ghost Aircraft) and high fidelity terrain concepts would favor simple guidance symbology (Pitch/Roll Flight Director or Unconnected Box Tunnel). Since there was no statistical evidence of an interaction between TPC and GSC, the results of the data analyses do not support these hypotheses. Perceived workload was lowest and subjective situation awareness was highest with the Tunnel with Ghost Aircraft GSC, for both approach and missed-approach scenarios, regardless of terrain portrayal concept (TPC). The fourth and final hypothesis stated that pilot technical performance would be improved with tunnel concepts. In terms of lateral and vertical flight technical error, the Tunnel with Ghost Aircraft GSC produced the smallest mean FTE, regardless of scenario or TPC. Furthermore, Photo Realistic terrain, regardless of GSC, resulted in the highest mean subjective SA scores while having no negative impact on FTE, for both the approach and missed-approach scenarios.

Interestingly, pilot category had a significant impact on perceived workload, SA, and L1 performance, for the missed-approach scenario and on perceived workload and SA for the approach scenario. During the approach scenario, VFR pilots who were unfamiliar with IMC flight were able to do as well as highly trained IFR pilots in terms of controlling flight path error (no significant L1 differences), and with less workload and more situation awareness, as a result of SVS. However, these results are troubling in that highly trained and experienced pilots reported having higher workload and less SA without a significant improvement in L1 performance over their less experienced counter parts. This outcome may simply be a result of dissimilar strategies employed by the different pilot categories when responding to the subjective SA and workload questionnaires or perhaps the intuitive nature of SVS truly does mitigate the perils that accompany the loss of out-the-window visibility for VFR pilots. However more research needs to be performed before a conclusion can be drawn in this regard.

\section{REMARKS}

This experiment was the first in a series of SD-HDD experiments designed to investigate the interaction of terrain and guidance concepts on a synthetic vision system primary flight display (SVS-PFD). Future SD-HDD experiments will focus on the enroute flight mode as well as the minification effects over a range of field of view sizes on the SVS-PFD.

\section{REFERENCES}

Charlton, S. (2002). Questionnaire Techniques for Test and Evaluation. In T.G. O’Brien \& S.G. Charlton (Eds.), Handbook of Human Factors Testing and Evaluation (pp. 225-246). Mahwah, NJ: Lawrence Erlbaum.

Endsley, M. (1999). Situation Awareness in Aviation Systems. In D.J. Garland, J.A.Wise, and V.D. Hopkin (Eds.), Handbook of Aviation Human Factors (pp. 257-276). Mahwah, NJ: Lawrence Erlbaum.

Glaab, L., and Hughes, M. (2003). Terrain Portrayal for Head-Down Displays Flight Test. $22^{\text {nd }}$ Annual Digital Avionics Systems Conference (DASC). Indianapolis, IN.

Hughes, M. and Glaab, L. and. (2003). Terrain Portrayal for Head-Down Displays Simulation Experiment Results. $22^{\text {nd }}$ Annual Digital Avionics Systems Conference (DASC). Indianapolis, IN.

Hummel, K., Landsberg, B., Roy, K., \& Wright, D. (2002). Nall Report 2002: AOPA Air Safety Foundation Accident Trends and Factors for 2001. AOPA Press. 